\title{
Vedolizumab Dose Escalation: In for a Penny, in for a Pound?
}

\author{
Robin J. Dart ${ }^{1,2} \cdot$ Peter M. Irving ${ }^{2,3} \cdot$ Mark A. Samaan $^{3}$ \\ Published online: 24 August 2020 \\ (c) Springer Science+Business Media, LLC, part of Springer Nature 2020
}

Regarding the choices of biologic agents for IBD, as is for all therapeutic interventions, it is often said that the most expensive drug is the one that fails to work. This is probably no truer than in the case of dose-escalated vedolizumab. There exists, therefore, a genuine desire within the IBD community to better understand exactly how effective dose escalation is and in which circumstances it is most likely to succeed. The desire to derive maximal benefit from vedolizumab is borne out of the fact that the number of approved therapies in IBD remains limited and that rates of non-response (primary and secondary) remain significant. For ulcerative colitis (UC) specifically, there are now six approved novel agents encompassing four different mechanisms of action (anti-TNF, antiIL12/23, anti-integrin, and Janus kinase (JAK) inhibition), a choice far more limited than those available for the treatment of rheumatoid arthritis, for example, which has at least twice as many advanced therapies available. Moreover, failure of medical therapy in UC generally necessitates life-altering surgery, in the form of colectomy and ileostomy formation. It, therefore, stands to reason that IBD clinicians should do everything in their power to extract the most from the currently available range of agents. Marching in lockstep with this aspiration is the necessity for IBD researchers to generate data informing their optimal use.

In this issue of Digestive Diseases and Sciences, Perry and colleagues [1] reported a retrospective study designed to investigate the observational effectiveness of vedolizumab dose escalation in a group of patients with UC. Amongst their cohort of 22 patients treated with vedolizumab with a partial response to standard 8-weekly dosing, 10 (45.5\%)

Mark A. Samaan

markasamaan@gmail.com

1 Department of Gastroenterology, Royal Free Hospital, London, UK

2 School of Immunology and Microbial Sciences, King's College London, London, UK

3 Department of Gastroenterology, St Thomas' Hospital, Guy's \& St Thomas', First Floor College House, North Wing, Westminster Bridge Road, London SE1 7EH, UK were observed to achieve remission (partial Mayo score (pMayo) of 0 or 1) upon dose escalation to 4-weekly, with the overall average pMayo score of the group falling from 4.4 at the point of escalation to 1.7 by the end of follow-up $(p<0.0005)$. These findings are broadly in keeping with a metanalysis of 4 studies (comprising 2 full articles and 2 abstracts), with a combined cohort of 111 patients with IBD, that demonstrated a random-effects pooled efficacy rate of $53.8 \%$ [2]. They are also in keeping with our own published cohort of 36 dose-escalated patients (18 Crohn's disease (CD), $18 \mathrm{UC}$ ), in which a $50 \%$ response rate was observed [3]. Moreover, rates of week 28 response to dose intensification for CD and UC reported as part of the GEMINI longterm extension were also similar at 17/32 (54\%) and 31/57 (53\%), respectively.

Perry and colleagues [1] also investigated factors that were associated with the need for dose escalation, demonstrating that both prior anti-TNF exposure and severe disease activity at baseline were independent predictors [1]. This type of detail clearly has the potential to impact clinical treatment algorithms, raising the question as to whether patients falling into these groups should receive 4-weekly maintenance dosing by default. It also makes one consider whether, at any point, there could be a change in labelling to allow for the optional/additional week 10 induction dose in UC in order to mirror that of CD.

Although the findings described here go some way to providing answers to clinically relevant questions, they raise several others. For example, and perhaps most importantly, why does dose escalation work at all? Why does prior antiTNF exposure and/or more severe disease at baseline predict the need for escalation? Which patients are most likely to respond to escalation? How does therapeutic drug monitoring (TDM) fit in (if at all)? Is de-escalation possible? How does subcutaneous administration affect vedolizumab's pharmacokinetics and pharmacodynamics?

If the hypothesis that vedolizumab largely acts via interrupting lymphocyte trafficking to the gut were true, the fact that $\alpha 4 \beta 7$ receptor saturation on $T$ lymphocytes in the blood and mucosa is near universal in treated patients might 
suggest that dose escalation is unlikely to work [4]. Yet, this simplistic model belies the complexity of the immune system, with $\alpha 4 \beta 7$ expressed on myeloid, B and innate lymphocytes as well as on conventional $\mathrm{T}$ cells. That mucosal $\alpha 4 \beta 7$ expression is a potential biomarker of response to vedolizumab, suggests that rather than simply inhibiting ingress to the gut, there may be a hitherto undefined local mechanism of action [5].

Other clues as to unanticipated mechanisms of action of vedolizumab can be found in a study of oral cholera vaccination, which demonstrated an anticipated partial inhibition of mucosal IgA responses and an unanticipated inhibition of serum IgG responses, which is not the case in the $\beta 7^{-/-}$mouse, and is not well explained by simple inhibition of lymphocyte homing to the intestine [6]. Furthermore, evidence derived from whole transcriptomes from patients treated with vedolizumab demonstrates significant changes in innate immune pathways, questioning the mechanistic assumptions underlying vedolizumab therapy [7]. Finally, vedolizumab not only blocks the $\alpha 4 \beta 7$ receptor but also promotes its internalisation, and while limited studies of effector function have not discerned clear functional consequences of this, the possibility of integrin signalling cannot yet be wholly dismissed [5]. Therefore, despite complete receptor occupancy as previously described, increased bioavailability of drug may be essential for maintaining other, as yet unappreciated therapeutic mechanisms.

Why prior anti-TNF exposure is associated with reduced efficacy of vedolizumab remains largely unknown, yet such patients are more likely to require dose escalation. It is possible that primary resistance to anti-TNF may in some cases be pre-ordained as in the case of those expressing high levels of oncostatin M [8]. Nevertheless, given that some develop secondary loss of response to anti-TNF, the question of whether exposure to anti-TNF may materially modify the immunopathology of IBD remains largely unexplored. Furthermore, it may be that non-response to anti-TNF is simply associated with disease severity. Decreased mucosal drug concentration could be due to well recognised anti-TNF loss in stool in severe UC combined with increased drug permeability through the injured mucosa, which in turn could hinder drug efficacy if elevated local mucosal concentrations indeed are needed for a full response. Investigation is currently underway in order to identify whether this phenomenon is indeed a significant determinant of vedolizumab pharmacokinetics [9].

Regarding TDM, initial data suggest that week 6 trough levels $<18.5 \mathrm{ug} / \mathrm{ml}$ predict the need for subsequent dose escalation and that levels above this threshold correlate with higher rates of mucosal healing at 1 year [10]. Nevertheless, these thresholds remain unvalidated; until further data are available, dosing should be empirically adjusted based upon objective measures of disease activity rather than on TDM.
Given the financial implications of such a strategy, it would clearly be desirable to identify patients most likely to respond to escalation, thus allowing for a more personalised approach to treatment adjustment and judicious use of a high-cost drug. For example, there are some data that suggest that a lower CRP at the point of escalation may be favourable [3]. Nevertheless, more and more specific predictors and biomarkers are badly needed. Indeed, if the controversies around the health economics of dose escalation were ever in doubt, one just needs to consider the case of the patient reported by Perry et al. [1], who was denied access by their insurance company. This is certainly not exclusive to insurance-based healthcare systems since it also occurs in single-payer systems, such as the UK's National Health Service, where access to dose escalation remains variable. Perhaps this issue will be partially resolved by recent data demonstrating that once remission has been achieved on 4-weekly dosing, it can be effectively maintained upon deescalation back to 8-weekly; out of 167 de-escalated patients in the GEMINI extended access program, only 6 required re-escalation over 56 weeks of follow-up.

It is unclear exactly how the newly available, subcutaneously administered preparation of vedolizumab will affect this field. Although there is no evidence of improved efficacy, one may reasonably expect the pharmacokinetic profile to be superior to that of intravenous administration, thus reducing the need for dose escalation from the standard 2-weekly injections. Indeed, escalation has neither been studied nor approved for the subcutaneous preparation and is likely to be prohibitively expensive.

In conclusion, until the necessary understanding to use an evidence-based, personalised approach to vedolizumab dose escalation, it appears as though it should be at least considered and perhaps trialled in the majority of patients failing standard dosing intervals. It would therefore seem that an appropriate clinical mindset when initiating vedolizumab therapy, would be 'in for a penny, in for a pound'.

\section{Compliance with Ethical Standards}

Conflict of interest R J Dart: Served as a consultant for Gamma Delta Therapeutics and Takeda. P M Irving: Served as a speaker, a consultant and/or an advisory board member for Abbvie, Warner Chilcott, Ferring, Falk Pharma, Takeda, MSD, Johnson and Johnson, Shire, Vifor Pharma, Pharmacosmos, Topivert, Genentech, Hospira, Samsung Bioepis, and has received research funding from MSD, Takeda and Pfizer. M A Samaan: Served as a speaker, a consultant and/or an advisory board member for Sandoz, Janssen, Takeda, MSD, Falk, Samsung Bioepis. 


\section{References}

1. Perry C, Fischer K, Elmoursi A, et al. Vedolizumab dose escalation improves therapeutic response in a subset of patients with ulcerative colitis. Dig Dis Sci. (Epub ahead of print). https://doi. org/10.1007/s10620-020-06486-x.

2. Peyrin-Biroulet L, Danese S, Argollo M, et al. Loss of response to vedolizumab and ability of dose intensification to restore response in patients with Crohn's disease or ulcerative colitis: a systematic review and meta-analysis. Clin Gastroenterol Hepatol. 2019;17:838-846.

3. Samaan MA, Birdi S, Morales MS, et al. Effectiveness of vedolizumab dose intensification to achieve inflammatory bowel disease control in cases of suboptimal response. Frontline Gastroenterol. 2020;11:188-193.

4. Ungar B, Kopylov U, Yavzori M, et al. Association of vedolizumab level, anti-drug antibodies, and alpha4beta7 occupancy with response in patients with inflammatory bowel diseases. Clin Gastroenterol Hepatol. 2018;16:697.e7-705.e7.

5. Rath T, Billmeier U, Ferrazzi F, et al. Effects of anti-integrin treatment with vedolizumab on immune pathways and cytokines in inflammatory bowel diseases. Front Immunol. 2018;9:1700.

6. Wyant T, Leach T, Sankoh S, et al. Vedolizumab affects antibody responses to immunisation selectively in the gastrointestinal tract: randomised controlled trial results. Gut. 2015;64:77-83.
7. Zeissig S, Rosati E, Dowds CM, et al. Vedolizumab is associated with changes in innate rather than adaptive immunity in patients with inflammatory bowel disease. Gut. 2019;68:25-39.

8. West NR, Hegazy AN, Owens BMJ, et al. Oncostatin M drives intestinal inflammation and predicts response to tumor necrosis factor-neutralizing therapy in patients with inflammatory bowel disease. Nat Med. 2017;23:579-589.

9. Investigation of the faecal loss of vedolizumab and its role in influencing serum drug levels, outcomes and response in ulcerative colitis. EudraCT number: 2018-002794-21. https://www.clini caltrialsregister.eu/ctr-search/trial/2018-002794-21/GB.

10. Ward MG, Sparrow MP, Roblin X. Therapeutic drug monitoring of vedolizumab in inflammatory bowel disease: current data and future directions. Ther Adv Gastroenterol. 2018;11:1756284818772786.

Publisher's Note Springer Nature remains neutral with regard to jurisdictional claims in published maps and institutional affiliations. 